\title{
Modified restoration technique for improved visual perception of shallow underwater imagery
}

\author{
M. Dhana Lakshmi and S. Sakthivel Murugan* \\ Underwater Acoustic Research Laboratory, Department of Electronics and Communication Engineering, \\ Sri Sivasubramaniya Nadar College of Engineering, Kalavakkam, Chennai 603 110, India
}

\begin{abstract}
Images captured underwater often suffer from quality degradation such as low contrast, non-uniform illumination, etc. due to the attenuation and backscattering of light by suspended underwater particles. To overcome this, restoration-cum-enhancement techniques are necessary. Here we present the modified underwater light attenuation prior (MULAP) model using supervised linear regression model to restore the degraded image. The image formation model (IFM)based restoration depends on dual factors: background light and transmission map. Initially, datasets are collected on the close-range point-of-interest. Then, experimental analyses are carried out for those images using the IFM-based methods. For the above techniques, both subjective analysis and objective analysis are done by considering dual metrics such as universal quality index and visual information fidelity factor. Finally, the proposed MULAP shows overwhelming qualitative and quantitative results among other state-of-the-art techniques.
\end{abstract}

Keywords: Image formation model, restoration techniques, underwater imagery, visual perception.

AUTONOMOUS underwater vehicles (AUVs) and remotely operated vehicles (ROVs) are extensively used in the scrutiny of submarine pipeline, seabed mapping, underwater image analysis, etc. ${ }^{1}$. The key challenge for an underwater vehicle is the usage of high-end cameras. Due to the physical and chemical characteristics of the underwater environment, the captured images may be affected by poor visibility and uneven bright specks ${ }^{2}$. These artifacts can result in a distorted underwater image which affects image-based tasks such as object detection, tracking, classification, segmentation, etc. Thus, retrieval of clear information from underwater images can be done by efficient enhancement-cum-restoration techniques. Many researchers have worked on underwater image enhancement and restoration. Song et al. $^{3}$ proposed an effective underwater image enhancement method in combination with underwater image restoration and colour correction. The new underwater dark channel prior (NUDCP) model approximates the transmission map ${ }^{\mathrm{TM}}$ of the red channel

\footnotetext{
*For correspondence. (e-mail: sakthivels@ssn.edu.in)
}

of underwater images and underwater light attenuation prior (ULAP) model estimates the depth map of the image. Yang et ll $^{4}$ performed analysis using various dark channel prior (DCP) methods on different standard underwater image datasets which contained bluish, greenish, yellowish, offshore and deep-sea images. It was concluded that the reality of colour restoration and the amount of detail retained in the dark areas were not in line with the subjective evaluation. Islam et al. ${ }^{5}$ proposed a conditional generative adversarial network (GAN)-based underwater image enhancement model on paired and unpaired (of poor and good quality) images. Li et al. ${ }^{6}$ presented a weakly supervised colour transfer method (modified Cycle GAN) to correct colour distortion in underwater images. Banerjee et al. ${ }^{7}$ proposed the $\mathrm{RGB} \mathrm{YCbCr}$ processing (RYPro) method and compared it with grey world (GW), white patch (WP), Adobe Photoshop equalization (APE) and unsupervised colour correction method (UCM). The RYPro improves the contrast, luminance and visual quality of the images without any loss of visual information. Existing work focuses on the enhancement of hazed images using a deep neural network. Due to computational complexity and system requirements, the authors focused on the image formation model (IFM)based technique than the deep-based technique. On considering the optical imaging model, the visual quality improvement techniques can be classified into two: IFMbased image restoration methods ${ }^{8}$ and IFM-free image enhancement methods ${ }^{9-13}$. To the best of our knowledge, some of the issues are not addressed in previous works such as image restoration-cum-enhancement techniques for close-range point-of-interest (POI) datasets. This study addresses these issues using the modified underwater light attenuation prior (MULAP) technique. Qualitative (subjective) analysis and quantitative (objective) analysis are done for the enhanced images by considering university quality index (UQI) and visual information fidelity (VIF) metrics with state-of-the-art techniques.

\section{IFM-based image restoration technique}

The underwater image restoration technique helps develop the degradation model by analysing underwater optical 


\section{RESEARCH ARTICLES}

images and the properties of light propagation, then infers the key factors of the developed physical model from previous knowledge and finally retains the restored image. The IFM can be expressed as

$$
I^{s}(y)=z^{s}(y) r^{s}(y)+B^{s}\left(1-r^{s}(y)\right)
$$

where $s$ is the RGB colour channel, $I^{s}$ the image captured by the optical camera, $r^{s}$ the residual energy, $B$ is the homogeneous background light. $z^{s}(y), \quad r^{s}(y)$ and $B^{s}\left(1-r^{s}(y)\right)$ denote the transmission and backscattering components respectively. IFM-based restoration depends on dual factors: background light (BL) and transmission map $^{3}$. By considering the optical properties (light attenuation, haze effect, etc.), different prior-based methods are used for restoration.

\section{DCP-based image restoration}

DCP is a statistical prior based on methodology ${ }^{14}$. It is observed that the clear images can exhibit dark pixels in a square image patch and identified that at least a single colour channel has zero intensity pixel value. Due to the following factors, the low-intensity value can occur in the dark channel: shadows, colourful objects and dark objects. DCP can be defined as

$$
R^{\mathrm{dark}}(y)=\min _{x \in \varphi(y)}\left(\min _{s \in \mathrm{RGB}} R^{s}(x)\right),
$$

where $R^{s}$ is the image radiance of all colour channels and $\varphi(y)$ is the square patch centred at $y$. An enhanced image model can be defined as

$$
I(y)=R(y) \cdot t(y)+A(1-t(y)),
$$

where $y$ represents the pixel coordinates and $I$ denotes the image retained in a participating medium. It is valid to assume that the model is in homogeneous background light. The isolation of transmission in a local patch can be done by considering eqs (2) and (3). The transmission map, $\tilde{t}(y)$ can be estimated by applying the minimum operation on both sides

$$
\tilde{t}(y)=1-\min _{x \in \varphi(y)}\left(\min _{s \in \mathrm{RGB}} \frac{I(y)}{A}\right),
$$

$\tilde{t}(y)$ is the approximation of the transmission component and its performance can be affected by local patches.

\section{Underwater DCP-based image restoration}

Due to difficulty in capturing real underwater images in water-out environments, it is also difficult to estimate the statistical relationship of a dark channel in haze-free images. Though the dark channel works well, wavelength independence issues are not properly addressed. DCP fails due to the red channel and affects the transmission estimation. Thus, underwater DCP is applied only to the green $(\mathrm{G})$ and blue (B) channels. UDCP can be expressed as

$$
R^{\mathrm{UDCP}}(y)=\min _{x \in \varphi(y)}\left(\min _{s \in \mathrm{GB}} R^{s}(x)\right),
$$

The air-light constant $A$ is calculated by finding the highest pixel in the underwater dark channel. The spectral matting technique is used to improve the estimated transmission. Here, $\varphi(y)$ is a $15 \times 15$ square patch.

$$
\tilde{t}(y)=1-\min _{x \in \varphi(y)}\left(\min _{s \in \mathrm{GB}} \frac{I(y)}{A}\right) .
$$

Saturation information of blurred images was used to adjust $\mathrm{TM}$ to improve the artificial light areas and enhance the overall colour fidelity of the images. However, the restored images exhibit unnatural colour.

\section{Other prior-based image restoration techniques}

New optical model (NOM) uses UDCP to estimate the scattering rate and transmission of blue and green tones ${ }^{15}$. Then the effective light attenuation difference method is used to estimate the background light of an underwater scene. GBdehazingRCorrection (GBRC) removes the attenuation of blue-green channels from scattering and corrects the red channel ${ }^{16}$. To obtain the differences among the three colour channels, the maximum intensity of the red channel is compared with that of the green and blue channels as

$$
D(y)=\max _{x \in \varphi, s \in r} I^{s}(y)-\max _{x \in \varphi, s \in\{g, b\}} I^{s}(y) .
$$

$D(y)$ denotes the major transformations among three dissimilar colour channels. The green and blue channels of blur-free image can be obtained by

$$
J^{s}(y)=\frac{I^{s}(y)-B^{s}}{t(y)}, s \in\{g, b\}
$$

Here $B^{s}$ denotes the background light that can be estimated based on the fact that the red channel weakens much faster than green and blue channels in an underwater image $^{16}$. The red light absorption rate is difficult to obtain. With the assumption of grey world theory ${ }^{17}$, that the colour in each channel average to be grey, and then the red channel can be corrected by

$$
\frac{\operatorname{avg} R+\operatorname{avg} B+\operatorname{avg} G}{3}=0.5 \text {. }
$$


Image blurriness and light absorption (IBLA) is the depth estimation method for underwater images based on image blurriness and light absorption ${ }^{18}$. Let $I(y)$ be the input image filtered by the $k \times k$ spatial Gaussian filter. The image blurriness can be computed by

$$
P(y)=\frac{1}{n} \sum_{i=1}^{n}\left|I(y)-G^{r_{i}, r_{i}}(y)\right| .
$$

Then the max-filter is applied to find the rough blurriness and light absorption is corrected by the image formation model.

\section{Proposed methodology}

ULAP is based on underwater light attenuation ${ }^{19}$. It is an effective linear model to obtain the scene depth map, BL, and $\mathrm{TM}$ for RGB channels to retain the original radiance underwater. Light attenuation prior is the change between the high-intensity value of green and blue and the red intensity value per pixel is highly correlated to the difference of the scene depth. To estimate scene depth, the linear model can be defined as

$$
g(y)=\beta_{0}+\beta_{1} l(y)+\beta_{2} r(x)
$$

where $y$ denotes the pixel coordinates, $g(y)$ the underwater scene depth at point $y, l(y)$ the maximum green-blue intensity value and $r(x)$ is the red intensity value. Using supervised linear regression, the coefficients $\beta_{0}, \beta_{1}$ and $\beta_{2}$ can be learned from the reference trained model which contains 24 million depth points ${ }^{19}$. The samples are randomly partitioned in a $k$-fold cross-validation technique, i.e. part of the sample is assigned as validation points and the $k-1$ residual points are considered as training points and the swapping of the process is repeated. Using the constants $\beta_{0}=0.53214, \beta_{1}=0.51309$ and $\beta_{2}=-0.91066$, the model can be defined to restore the depth maps. The background light $B_{l}^{s}$ can be estimated by considering the maximum intensity value after removal of the $0.1 \%$ distant point. This is because the foreground object pixel contains the brighter (higher) pixel values than the background pixel. The transmission component can be computed from the actual scene depth map $d_{\mathrm{m}}(y)$ it is defined as

$$
d_{\mathrm{m}}(y)=S_{\mathrm{c}} \times g(y) .
$$

Here $S_{\mathrm{c}}$ is a scaling constant. Then the transmission map is estimated as

$$
t_{\lambda}(y)=\operatorname{rgr}(\lambda)^{d_{\mathrm{m}}(y)}
$$

Here, $r g r$ is assigned as $0.83,0.95$ and 0.97 . The scene radiance can be computed from background light $B_{l}^{s}$ and transmission map $t_{m}(y)$ as follows

$$
S_{r}=\frac{1}{\min \left(\max \left(t_{\lambda}(y) 0.1\right), 0.9\right)}\left(I^{s}(y)-B_{l}^{s}\right)+B_{l}^{s} .
$$

Though the image is well restored, it suffers from contrast completeness. So, the image undergoes an enhancement technique called automated multi-scale retinex with colour restoration ${ }^{21}$. Basically, the retinal theory is defined as

$$
\text { Retinex }=R(y) * L(y)
$$

where $R$ denotes the reflectance component and $L$ denotes the illuminance. The illumination can be done with a lowpass filter. Then multi-scale retinex is defined as

$$
\operatorname{MSR}(y)=\sum_{m=1}^{M} w_{m} y_{m j}
$$

Here, $M$ is the number of scales, $y_{m i}$ the $j$ th component of the $m$ th scale and $w_{m}$ is the assigned weight. Though the image is enhanced, it may have halo-artifacts. Hence the colour restoration in multi-scale retinex can be done by

$$
\begin{aligned}
& \operatorname{MSRCR}_{\text {automated }}=G\left[C_{j}(y) \operatorname{MSR}(y)+d\right], \\
& C_{j}(y)=f\left[I_{j}^{\prime}(y)\right] .
\end{aligned}
$$

$C_{i}(y)$ is a function for the colour restoration factor and $G$ is the gain value ${ }^{20}$. Finally, the enhanced image is obtained from the restored image with the complete information.

$$
\begin{aligned}
& C_{j}(y)=\mu \log \left[\lambda I_{j}^{\prime}(y)\right], \\
& C_{j}(y)=\mu \log \left[\lambda I_{j}^{\prime}(y)\right]-\mu \log \left[\sum_{j=1}^{S} I_{m}^{\prime}(y)\right] .
\end{aligned}
$$

Here $\mu$ is the improvement constant and $\lambda$ deals with the nonlinearity issue of the algorithm. $G$ and $d$ are the absolute gain factor and offsets respectively. The values are obtained by computation of variance and histogram$\operatorname{plot}^{20}$. Figure 1 shows the workflow of MULAP.

\section{Results and discussion}

Due to the properties of the underwater environment, the acquired images can be affected by poor visibility, absorption and scattering of light. Thus, restoration-cumenhancement techniques are required for the degraded images. The datasets 'I1 to I5' were captured in closerange (CR) POI on 10 April 2019 from $12^{\circ} 04^{\prime} 54.3^{\prime \prime} \mathrm{N}$, $79^{\circ} 41^{\prime} 11.2^{\prime \prime} \mathrm{E}$ at a depth of $50 \mathrm{~m}$. Using a low-light HD 


\section{RESEARCH ARTICLES}

USB camera, data were collected at different depths from 5 to $15 \mathrm{~m}$ in the shallow area. To test the adaptiveness of the algorithm, sample 'I6' from the SUN standard dataset was also considered ${ }^{20}$. The proposed technique was compared with IFM-based techniques such as GBRC, NOM, UDCP and IBLA. Then qualitative (subjective) analysis and quantitative (objective) analysis were carried out (Table 1).

\section{Quantitative analysis}

Quantitative analysis was carried out to quantify information, noise and similarity of underwater images. The metrics used were UQI and VIF.

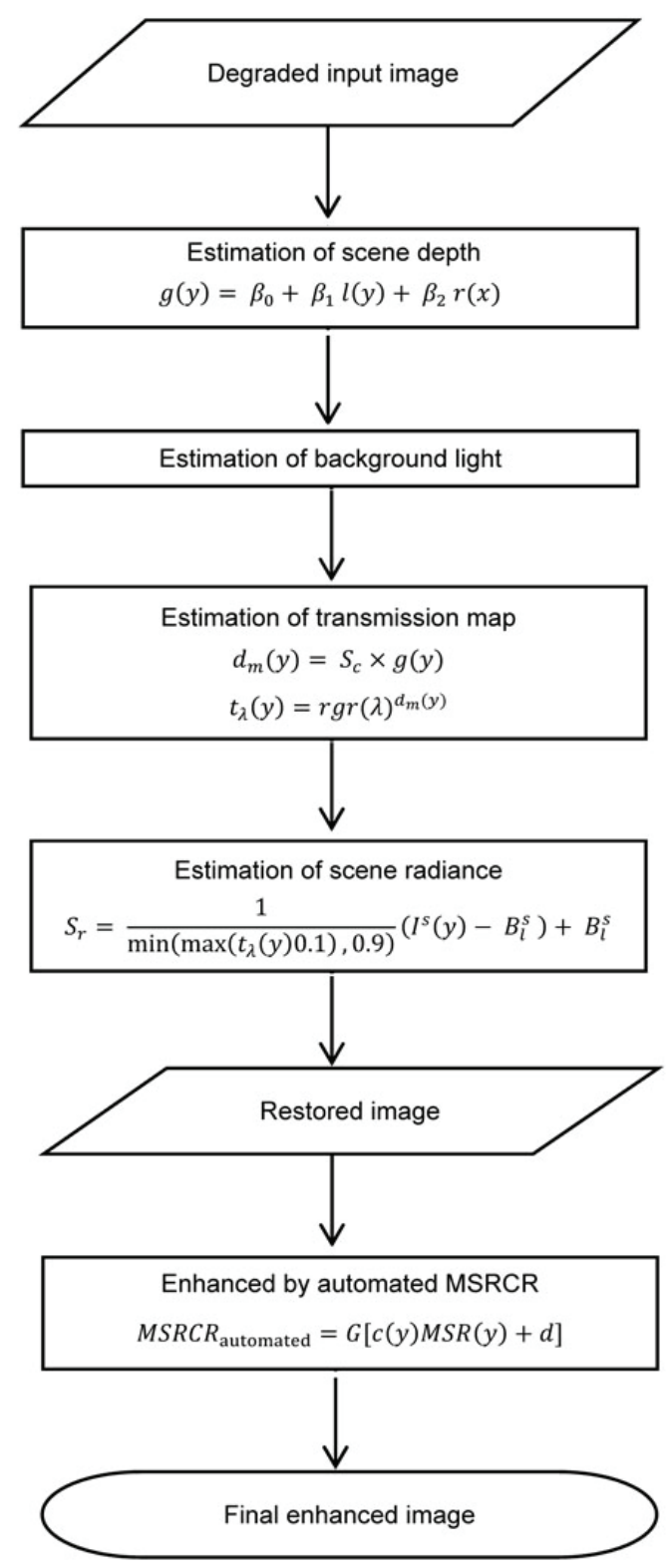

Figure 1. Workflow of the modified underwater light attenuation prior model.

\section{$U Q I$}

This is the measure of the loss of correlation, luminance distortion and contrast distortion ${ }^{22}$.

$$
\mathrm{UQI}=\frac{1}{M} \sum_{j=1}^{M} \frac{4 \sigma_{x y} \bar{x} \bar{y}}{\left(\sigma_{x}^{2}+\sigma_{y}^{2}\right)\left[(\bar{x})^{2}+(\bar{y})^{2}\right]} .
$$

Let $x$ and $y$ be the original blurred image and enhanced image respectively. UQI can be computed in $M$ steps within the sliding window moves pixel-by-pixel in both horizontal and vertical direction until bottom-right corner pixel is reached. For optimal UQI, the computed value must be high.

\section{$V I F$}

This is the image quality measure to evaluate the natural scene statistics as follows ${ }^{23}$

$$
\mathrm{VIF}=\frac{\left.\left.\sum_{j \in \text { subbands }} I(C ; F) \mid S\right)\right)}{\left.\left.\sum_{j \in \text { subbands }} I(C ; E) \mid S\right)\right)} .
$$

Table 1 shows the quantitative evaluation of IFM-based image restoration techniques. It is observed that the proposed technique gives a higher score and proves to retain the information well. Other techniques blindly intensify the unwanted information, i.e. noises and show lower scores. Compared to them, MULAP achieves the best assessment in terms of VIF and UQI. Table 1, also shows that the total running time of the proposed method is much lower than the others. This proves that the IFMbased methods can retain the actual scene but take more time to compute the optical parameters, while MULAP can rapidly enhance the images. Overall, the proposed technique enhances the images with good contrast, complete information and low time complexity.

\section{Qualitative analysis}

Underwater image restoration/enhancement is necessary to improve the visual clarity of blurred images and expose complete information, for the purpose of feature extraction and computer vision analysis. Figure 2 shows the qualitative results of the proposed and other image restoration methods. The image enhanced by the NOM method shows a large amount of red tone compared to the original image. GBRC and NOM do not perform the blind redistribution of pixels. Both methods are based on adaptive constraints to prevent overall histogram stretching. Thus their results are over-enhanced. GBRC and NOM restore the image by overwhelming of $\mathrm{R}$ component and 
RESEARCH ARTICLES

Table 1. Quantitative analysis of the proposed technique with other techniques

\begin{tabular}{llllllc}
\hline Metric & \multicolumn{1}{c}{ Image } & UDCP & NOM & GBRC & IBLA & Proposed \\
\hline UQI & I1_WHOLE_TYRE & 0.2387 & 0.5811 & 0.5169 & 0.5835 & 0.8043 \\
& I2_FERN & 0.5629 & 0.5714 & 0.5175 & 0.7757 & 0.7940 \\
& I3_PARTIAL_TYRE & 0.4260 & 0.6362 & 0.4877 & 0.5377 & 0.7885 \\
& I4_DIVER & 0.1124 & 0.5671 & 0.4559 & 0.1871 & 0.7029 \\
& I5_ROCK & 0.4703 & 0.5369 & 0.4172 & 0.4454 & 0.7811 \\
& I6_FISH & 0.5785 & 0.5361 & 0.4980 & 0.7006 & 0.7165 \\
VIF & & & & & \\
& I1_WHOLE_TYRE & 0.8134 & 0.6799 & 1.0144 & 1.2869 & 1.288 \\
& I2_FERN & 1.2548 & 0.7508 & 1.0430 & 1.1160 & 1.262 \\
& I3_PARTIAL_TYRE & 0.9220 & 0.5908 & 0.8558 & 0.8920 & 0.9769 \\
& I4_DIVER & 0.6784 & 0.8933 & 0.9143 & 0.7117 & 0.9529 \\
& I5_ROCK & 1.3676 & 0.8585 & 0.9366 & 1.3214 & 1.3951 \\
& I6_FISH & 0.8752 & 0.5013 & 0.7836 & 0.7430 & 0.8886 \\
\multicolumn{1}{c}{ Total running time (min) } & 3.48 & 8.13 & 5.49 & 8.42 & 1.16 \\
\hline
\end{tabular}
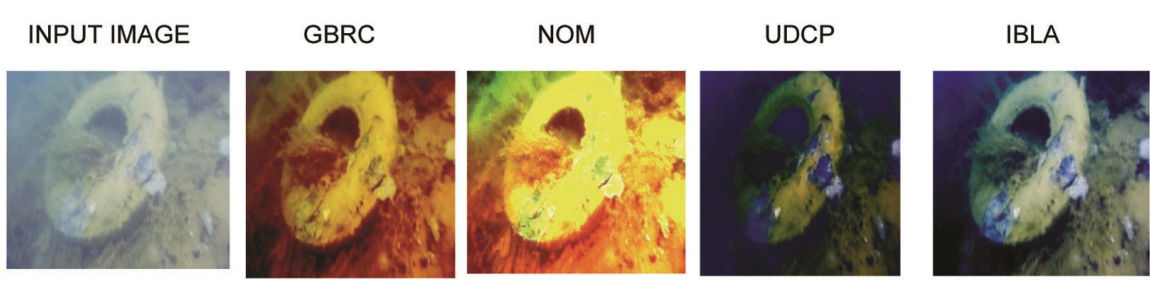

PROPOSED

\section{I1_WHOLETYRE}
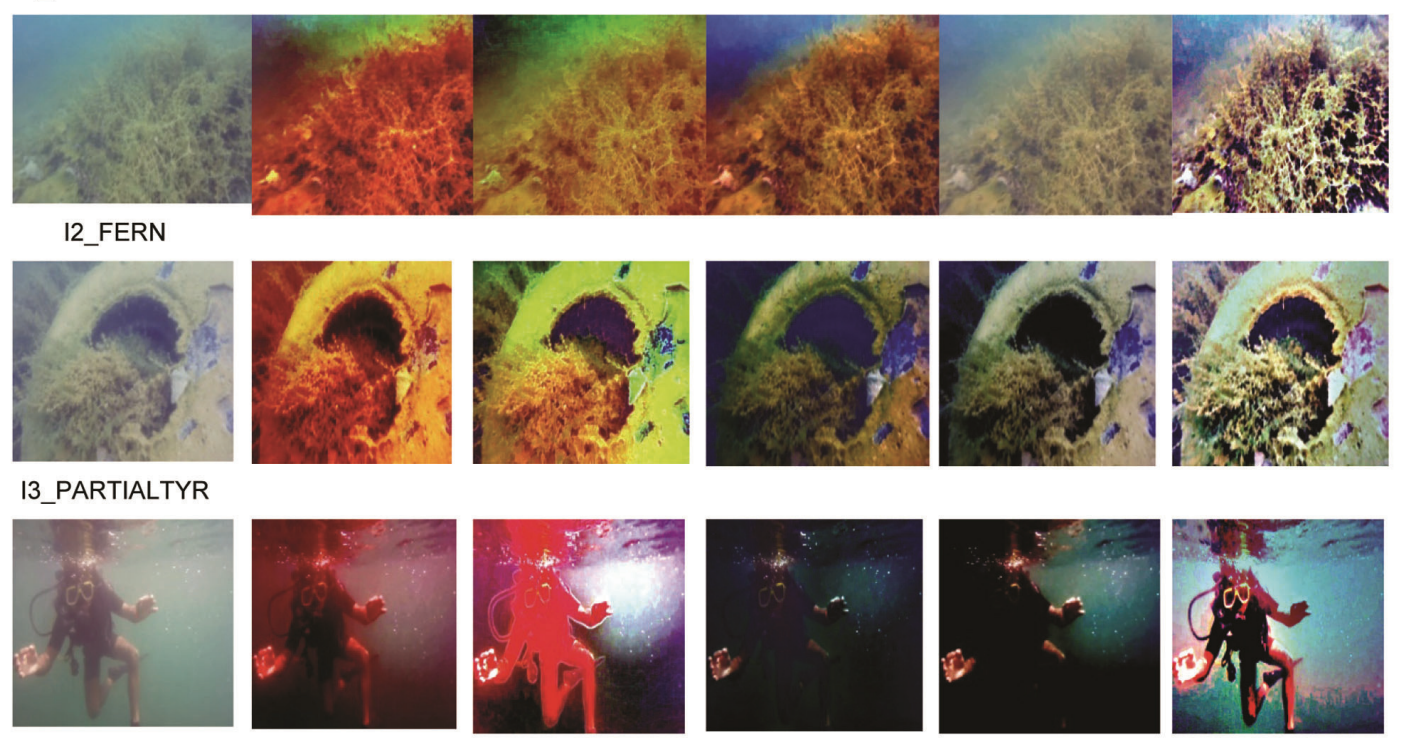

14_DIVER
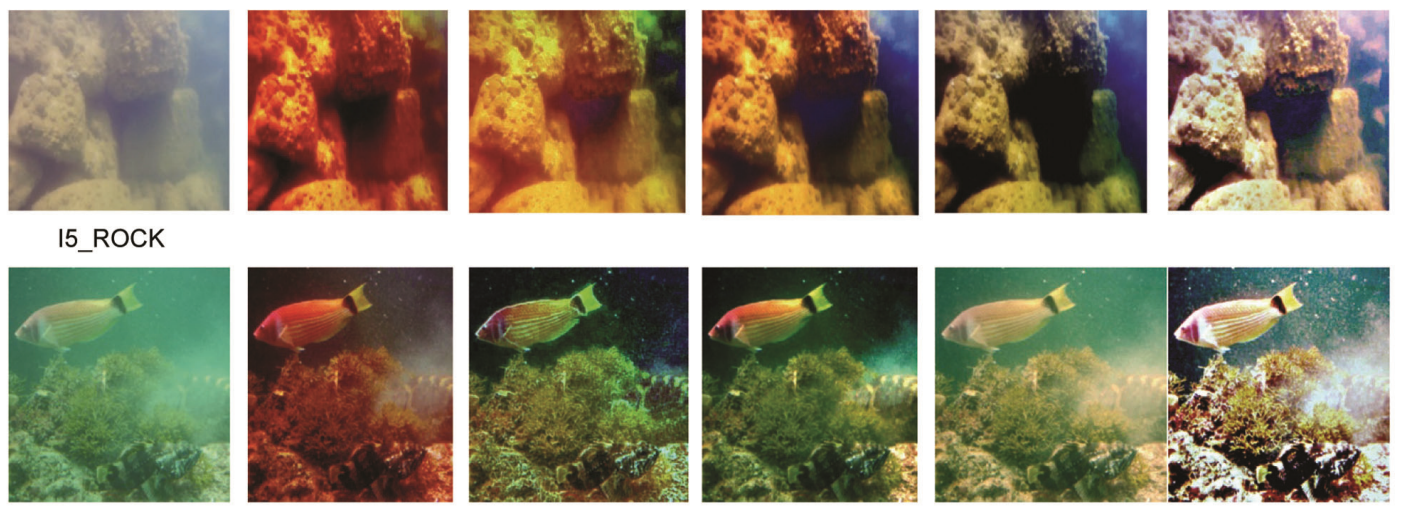

16_FISH

Figure 2. Qualitative comparison of the proposed technique with other techniques. 


\section{RESEARCH ARTICLES}

produce enormous noise and distortion. MULAP builds a strong relationship between the RGB channels and gives the best restoration results compared to the others. It also retains the edge and texture information in a clear manner. Comprehensively, MULAP achieves superior performance compared to the other techniques.

\section{Conclusion}

This study presents the MULAP model with retinex and analyses the experiment-based comparison of image visibility improvement methods using quality evaluation metrics (UQI and VIF). It then provides a discussion on the shortcomings of each of these techniques. IFM-based methods can retain the actual scene but takes more time to compute the optical parameters, while MULAP can rapidly enhance the images. It also improves the visibility of the hidden structures compared to the source. Overall, this study outlines the challenges and issues of the quality improvement methods using both qualitative and quantitative evaluation. MULAP has achieved better quantitative results among the image enhancement and restoration techniques. It is also noticed that NOM and GBRC resultants are over-enhanced which are against the subjective preference to naturalness. Hence, future research can be carried out using networks with low complexity to achieve a smart combination of both qualitative and quantitative assessments to improve the quality of underwater images with complete edge details.

1. Mangeruga, M., Cozza, M. and Bruno, F., Evaluation of underwater image enhancement algorithms under different environmental conditions. J. Mar. Sci. Eng., 2018, 6, 10.

2. Huang, D., Wang, Y., Song, W., Sequeira, J. and Mavromatis, S., Shallow-water image enhancement using relative global histogram stretching based on adaptive parameter acquisition. In Proceedings of International Conference on Multimedia Modeling, Springer, 2018

3. Wei, S., Wang, Y., Huang, D., Liotta, A. and Perra, C., Enhancement of underwater images with statistical model of background light and optimization of transmission map. IEEE Trans. Broadcast., 2020, 66, 153-169.

4. Miao, Y., Hu, J., Li, C., Rohde, G., Du, Y. and Hu, K., An in-depth survey of underwater image enhancement and restoration. IEEE Access, 2019, 7, 123638-123657.

5. Islam, Md J., Xia, Y. and Sattar, J., Fast Underwater Image enhancement for improved visual perception. IEEE Robot. Automat. Lett., 2020, 5, 3227-3234.

6. Li, C., Guo, J. and Guo, C., Emerging from water: underwater image color correction based on weakly supervised color transfer. IEEE Signal Proc. Lett., 2018, 25, 323-327.

7. Banerjee, J., Ray, R., Vadali, S. R. K., Shome, S. N. and Nandy, S., Real-time underwater image enhancement: an improved approach for imaging with AUV-150. Sadhana, 2016, 41, 225-238.

8. Wang, Y., Song, W., Fortino, G., Qi, L.-Z., Zhang, W. and Liotta, A., An experimental-based review of image enhancement and image restoration methods for underwater imaging. IEEE Access, 2019, 7, 140233-140251.

9. Dorothy, R., Joany, R. M., Joseph Rathish, R., Santhana Prabha, S. and Rajendran, S., Image enhancement by histogram equalization. Int. J. Nano. Corr. Sci. Eng., 2015, 2, 21-30.

10. Ma, J., Fan, X., Yang, S. X., Zhang, X. and Zhu, X., Contrast limited adaptive histogram equalization based fusion for underwater image enhancement. Int. J. Pattern Recognit. Artif. Intell., 2018, 32, 1854018.

11. Iqbal, K., Salam, R. A., Osman, A. and Talib, A. Z., Underwater image enhancement using an integrated colour model. IAENG Int. J. Comput. Sci., 2007, 34.

12. Iqbal, K., Odetayo, M., James, A., Salam, R. A. and Talib, A. Z. H., Enhancing the low quality images using unsupervised colour correction method. In Proceedings of 2010 IEEE International Conference on Systems, Man and Cybernetics, IEEE, 2010.

13. Ghani, A., Abdul, S. and Isa, N. A. M., Underwater image quality enhancement through composition of dual-intensity images and Rayleigh-stretching. SpringerPlus, 2014, 3(1), 757.

14. Drews, P., Nascimento, E., Moraes, F., Botelho, S. and Campos, M., Transmission estimation in underwater single images. In Proceedings of the IEEE International Conference on Computer Vision Workshops, 2013.

15. Wen, H., Tian, Y., Huang, T. and Gao, W., Single underwater image enhancement with a new optical model. In 2013 IEEE International Symposium on Circuits and Systems (ISCAS2013), IEEE, 2013.

16. Li, C., Quo, J., Pang, Y., Chen, S. and Wang, J., Single underwater image restoration by blue-green channels dehazing and red channel correction. In Proceedings of 2016 IEEE International Conference on Acoustics, Speech and Signal Processing (ICASSP), IEEE, 2016.

17. Ebner, M., Color constancy based on local space average color. Mach. Vision Appl., 2009, 20, 283-301.

18. Peng, Y.-T. and Cosman, P. C., Underwater image restoration based on image blurriness and light absorption. IEEE Trans. Image Proc., 2017, 26, 1579-1594.

19. Song, W., Wang, Y., Huang, D. and Tjondronegoro, D., A rapid scene depth estimation model based on underwater light attenuation prior for underwater image restoration. In Proceedings of Pacific Rim Conference on Multimedia, Springer, 2018.

20. Parthasarathy, S. and Sankaran, P., An automated multi scale retinex with color restoration for image enhancement. In Proceedings of 2012 National Conference on Communications (NCC), IEEE, 2012.

21. Xiao, J., Hays, J., Ehinger, K. A., Oliva, A. and Torralba, A., Sun database: Large-scale scene recognition from abbey to zoo. In 2010 IEEE Computer Society Conference on Computer Vision and Pattern Recognition, IEEE, 2010.

22. Wang, Z. and Bovik, A. C., A universal image quality index. IEEE Signal Proc. Lett., 2002, 9, 81-84.

23. Wang, Z., Alan, B., Hamid, S. and Eero, S., Image quality assessment: From error visibility to structural similarity. IEEE Trans. Image Proc., 2004, 13, 600-612.

ACKNOWLEDGMENTS. This work is part of a project funded by the Department of Science and Technology, New Delhi under State Science and Technology Programme (File No: DST/SSTP/TN/102/ 2017-18). We thank Mr A. Muthuvel for data support.

Received 11 January 2021; revised accepted 26 April 2021

doi: $10.18520 / \mathrm{cs} / \mathrm{v} 121 / \mathrm{i} 1 / 103-108$ 